\title{
Determinants of hypoxemia in children associated with pneumonia
}

\author{
Pramila Ramawat ${ }^{1}$, Balkishan Sharma ${ }^{2}$ \\ ${ }^{1}$ Senior Resident, Department of Pediatrics, Modern Institute of Medical Sciences, Indore, India, ${ }^{2}$ Associate Professor (Biostatistics), \\ Department of Community Medicine, Sri Aurobindo Medical College and P. G. Institute, Indore, India
}

Background and Objectives: The most severe manifestation of pneumonia is hypoxemia has been shown to be a risk factor for morbidity and mortality. Authors investigated associating factors and determinants of hypoxemia in children with pneumonia. Materials and Methods: A crosssectional study is designed among children of pediatric outpatient and emergency department that enrolled at Government Multi-Speciality Hospital, Chandigarh. One hundred fifty children recruited for study. The demographic and clinical parameters were recorded. Oxygen saturation measured by pulse oximeter. Results: The prevalence of hypoxemia was $48 \%$ and 61 (84.7\%) infants aged 1 year found with hypoxemia. Age $(p=0.006)$, respiratory rate $(p=0.001)$ and severity of pneumonia $(p=0.001)$ were strongly associated with hypoxemia. The prevalence of severe and very severe pneumonia among hypoxemic were $56.1 \%$ and $73.7 \%$ respectively. Central cyanosis (98.7\%), peripheral (98.7\%) cyanosis, head nodding $(97.4 \%)$ grunting $(96.15 \%)$ were highly specific but suprasternal $(62.82 \%)$, subcostal $(43.58 \%)$ and intercostal retractions $(44.87 \%)$ were fairly specific sign. Sensitivity for subcostal $(81.94 \%)$ and intercostal retractions $(83.33 \%)$ was very high but was fair for intercostal $(83.33 \%)$ retraction. Grunting $(p=0.009)$, nasal flaring $(p=0.008)$, subcostal $(p=0.001)$ and intercostal $(p=0.000)$ retractions were strongly but suprasternal retraction was significantly $(p=0.024)$ associated with hypoxemia. Dyspnea $(97.22 \%)$ was very sensitive while decrease feeding $(84.61 \%)$ and irritability $(83.33 \%)$ was highly but lethargy $(58.97 \%)$ was fairly specific symptom. Conclusions: Study suggested that clinical signs and symptoms such as chest wall retraction, decrease feeding, dyspnea, grunting and nasal flaring in children with pneumonia may be utilized as markers for hypoxemia in conditions where pulse-oximeter isn't available. This study supports the view of hypoxemia was disabling factor in better functional recovery in severity of pneumonia.

Access this article online Website:

http://nepjol.info/index.php/AJMS DOI: 10.3126/ajms.v7i2.13049 E-ISSN: 2091-0576 P-ISSN: 2467-9100

Key words: Hypoxemia, Pneumonia, Pulse-oximeter, Sensitivity, Specificity

\section{INTRODUCTION}

Hypoxemic children with pneumonia are five times more likely to die than those without hypoxemia. ${ }^{1}$ Hypoxemia, the most severe manifestation of pneumonia, has been shown to be a risk factor for mortality. Acute lower respiratory tract illness is a leading cause of hospitalization in children, followed by diarrhea, in developing countries. ${ }^{2}$ Pneumonia is an illness of lower respiratory tract in which the lungs become inflamed and congested and alveolar spaces are filled with fluid and cells-polymorphs and lymphocytes. Pneumococus and Hemophilus influenzae are common bacteria causing pneumonia in children. Pneumonia affects individuals of all ages but occur more frequently in children and the elderly but deaths due to it are also preventable by immunization and access to simple effective treatments. ${ }^{3}$ An estimated 146-159 million children suffer from pneumonia every year worldwide. ${ }^{4}$ According to WHO $13 \%$ of deaths in children aged 1 to 4 years are because of pneumonia in India. ${ }^{5}$

The association between hypoxemia with pneumonia and high mortality suggests that early detection and management of hypoxemia are important aspects in 
children with pneumonia. Assessment of hypoxemia in pneumonia has become possible due to pulse oximeter which is a useful and reliable predictor of hypoxemia in pneumonia, ${ }^{6}$ and is a non invasive and simple method of measuring oxygen saturation. ${ }^{7}$ A pulse oximeter, however, is expensive and hence clinical signs and symptoms that best predict hypoxemia in pneumonia have evaluated in earlier studies in other developing countries. Clinical features like cyanosis, tachypnea, grunting, head nodding, inability to cry, no spontaneous movement during clinical examination and chest retractions have been identified as the best clinical predictors of hypoxemia ${ }^{1,8}$ and for the majority of children in developing countries the assessment is based upon clinical signs alone. ${ }^{9}$ Clinical signs and symptoms such as chest wall retraction, inability to feed, grunting and cyanosis may be used by health workers to allow rational use of oxygen in places where there is shortage of oxygen and pulse-oximeter is not available. ${ }^{10}$

The greatest potential to reduce pneumonia related deaths require investment in human resources and equipment for optimum management of hypoxemia. ${ }^{11}$ However, detection and effective management of hypoxemia plays an important role in management of hypoxemia in children with pneumonia. Studies have been done to correlate clinical signs and symptoms of pneumonia with hypoxemia. ${ }^{12}$ Case fatalities in children with pneumonia is associated with several factors including clinical condition of the child at the time of admission, quality of care and monitoring during hospital stay, and appropriate supportive care including oxygen therapy and ventilation. ${ }^{13}$ The understanding of the sign and or symptom of pneumonia with hypoxemia has evolved over the centuries has left many aspects of pneumonia with hypoxemia as the subject of discussion and research.

The authors hypothesized that pneumonia with hypoxemia is associated with various demographical and clinical sign and symptom during hospitalization among children. Therefore, the authors aimed to utilize the sign and symptom in pneumonia with hypoxemia in clinical condition to identify the associating factors and determinants of hypoxemia in pneumonic children.

\section{MATERIALS AND METHODS}

A cross-sectional study is designed among children of pediatric outpatient and emergency department of Government Multi-Speciality Hospital, Sector 16, Chandigarh that presented with an acute history of cough, fever and rapid or difficult breathing during the period from July to December 2008.
One hundred fifty infant and children between the ages of 2 months to 5 years recruited during specified period, selected as subjects according to inclusion-exclusion criteria, and categorized according to classification of pneumonia by world health organization (WHO). Subjects were eliminated from the study that had a case of no pneumonia as per WHO classification of pneumonia, known case of bronchial asthma, prominent wheeze, abnormal chest shape, cardiac illness and signs of other systematic diseases. A detailed present history taken by caregiver of subjects who satisfied inclusion criteria including complaint of cough, fever, difficulty in respiration, decreased feeding, and chest pain duration of symptoms and past history.

Detailed examination recorded at time of enrollment, according to a questionnaire prepared for the purpose of study. Child's general condition, presence of pallor, icterus, clubbing, edema, and lymphadenopathy noted. Respiratory rate and heart rate counted for 1 minute and temperature recorded after putting thermometer for 2 minute in axilla. Respiratory system examination included central and peripheral cyanosis, head nodding, grunting, nasal flaring, suprasternal, subcostal and intercostal retractions. Other systems such as cardio vascular, central nervous and gastrointestinal system examined. Oxygen saturation measured by pulse oximeter (Criticare -504 US) on middle/index finger/big toe, before starting treatment. $\mathrm{SaO}_{2}$ reading stable for 3 minutes noted. Hypoxemia defined as arterial oxygen saturation of $<90$ percentage recorded by pulse oximetry. Prior consent from the parents/guardians of children for the publication of the clinical details and others has taken into account. The anthropometric and demographic measurements recorded.

\section{Statistical analysis}

The data presented as percentages for categorical variables and mean with standard deviation (SD) for continuous variables. The Chi-square test carried out to identify the association of different clinical parameters and demographics with groups (non-hypoxemic and hypoxemic). The data we analyzed by using statistical software SPSS Version 10.0. The probability value, $\mathrm{p}<0.05$ was considered as significant while from $\mathrm{p}<0.01$ to $\mathrm{p}<0.001$ were considered as strongly/highly significant.

\section{RESULTS}

Out of 150 subjects, $78(52 \%)$ were reported no hypoxemic while $72(48 \%)$ were hypoxemic that diagnosed by pulse oximetry. Overall prevalence of hypoxemia was $48 \%$.

Table 1 presents the patient characteristics. Mean age (S.D.) of total children was 13.04 (12.61) months. Mean 
age (S.D.) of children without hypoxemia was significantly different from those who had hypoxemia $(15.44 \pm 14.22$ versus $10.44 \pm 10.05)$. There were 19 (24.4\%) female and $59(75.6 \%)$ male children found without hypoxemia as compared to 27 (37.5\%) female and 45 (62.5) male children had hypoxemia but the gender differences in both the groups were statistically insignificant $(\mathrm{p}=0.081)$ which is also shown by Figure 1. Out of total, 124 children were from low socioeconomic status while 26 had middle and no children from high status $(\mathrm{p}=0.053)$. Children without hypoxemia had significantly $(\mathrm{p}=0.014)$ different weight $(8.08 \pm 3.02$ kilogram) as compared to weight $(6.86 \pm 2.94$ kilogram) of those who had hypoxemia. The height was with in normal limit but weight less than $80 \%$ of expected was between $80-60 \%$ in 64 children in which 22 found without hypoxemia and 42 noted with hypoxemia. The weight was significantly associated $(\mathrm{p}=0.002)$ with hypoxemia (Table 1).

Table 2 shows that the children below 1 year of age found with hypoxemia more frequently than children aged $1-5$ years. $48(61.5 \%)$ children were below 1 year, $17(21.8 \%)$ between $1-2$ years and $13(16.7 \%)$ were aged $2-5$ years had no hypoxemia as compared to $61(84.7 \%), 6(8.3 \%)$ and $5(6.9 \%)$ children were aged 1 year, $1-2$ year and $2-5$ years respectively found with hypoxemia. Hypoxemia was strongly associated $(p=0.006)$ and impacted the age. Respiratory rate of more than 60 per minute (tachypnea) was present in 18 (34\%) children noted without hypoxemia as compared to $35(66 \%)$ children with hypoxemia. The respiratory rate was strongly associated with hypoxemia $(\mathrm{p}=0.001)$ was confirmed statistically.

The statistical agreement shows in Table 2 that hypoxemia was significantly impacted and strongly associated $(\mathrm{p}=0.001)$ with the severity of pneumonia. Prevalence of hypoxemia increased with increased severity of pneumonia.

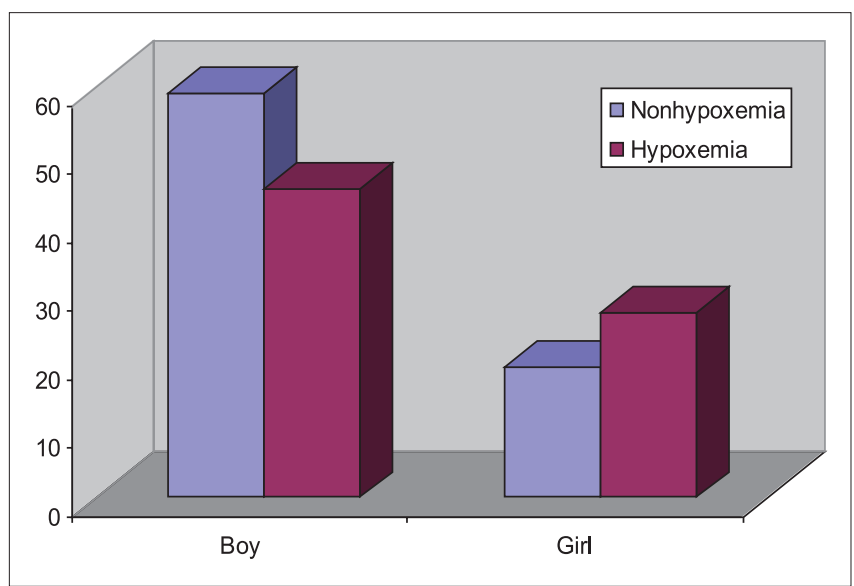

Figure 1: Gender wise distribution of subjects according to disease status.
Non-severe, severe, and very severe pneumonia was present in 55, 57, and 38 children respectively. 43 (78.2\%), $25(43.9 \%)$ and $10(26.3 \%)$ children had non-severe, severe and very severe pneumonia respectively had no hypoxemia as compared to $12(21.8 \%), 32(56.1 \%)$ and $28(73.7 \%)$ who had hypoxemia.

Table 3 reveals that central and peripheral cyanosis were present in $2.8 \%$ and $5.6 \%$ hypoxemic children respectively found with highly specific (98.7\%) sign while head nodding was in $5(6.9 \%)$ hypoxemic children but the differences in groups couldn't reach at statistical significance $(p=0.513$, $\mathrm{p}=0.0145, \mathrm{p}=0.204)$. Head nodding had high specificity (97.4\%) but low sensitivity (6.9\%).

\begin{tabular}{|c|c|c|c|}
\hline Parameter & $\begin{array}{c}\text { No hypoxemic } \\
78(52 \%)\end{array}$ & $\begin{array}{c}\text { Hypoxemic } \\
72(48 \%)\end{array}$ & $p$-value \\
\hline \multicolumn{4}{|l|}{ Age (months) } \\
\hline Mean \pm S.D & $15.44 \pm 14.22$ & $10.44 \pm 10.05$ & $p=0.015^{*}$ \\
\hline Range & $2-60$ & $2-48$ & \\
\hline \multicolumn{4}{|l|}{ Sex } \\
\hline Male/female & $59 / 19$ & $45 / 27$ & $p=0.081^{\otimes}$ \\
\hline \multicolumn{4}{|l|}{ Weight (kilogram) } \\
\hline Mean \pm S.D & $8.08 \pm 3.02$ & $6.86 \pm 2.94$ & $p=0.014^{*}$ \\
\hline Range & $2.5-16$ & $2.6-14$ & \\
\hline$<80 \%$ of expected & 22 & 42 & $\mathrm{p}=0.002^{\star \star *}$ \\
\hline \multicolumn{4}{|l|}{$\begin{array}{l}\text { Socioeconomic } \\
\text { status }(n(\%))\end{array}$} \\
\hline Low & $60(76.92)$ & $64(88.88)$ & $p=0.053^{*}$ \\
\hline Middle & $18(23.08)$ & $8(11.12)$ & \\
\hline High & - & - & \\
\hline
\end{tabular}

***Differences between groups were highly/strongly significant at the 0.002 level of significance. *Differences between groups were significant at the 0.05 and 0.02 levels of significance. ${ }^{\otimes}$ Differences weren't significant at the 0.05 level of significance

\begin{tabular}{|c|c|c|c|c|}
\hline \multirow[t]{2}{*}{ Parameters } & \multicolumn{2}{|c|}{ Groups (\%) } & \multirow{2}{*}{ Chi-Square } & \multirow[t]{2}{*}{$p$ value } \\
\hline & $\begin{array}{c}\text { No } \\
\text { hypoxemic }\end{array}$ & Hypoxemic & & \\
\hline \multicolumn{5}{|l|}{ Age (year) } \\
\hline Up to 1 & $48(61.5)$ & 61 (84.7) & 10.14 & $\mathrm{p}=0.006^{\star * *}$ \\
\hline$>1-2$ & $17(21.8)$ & $6(8.3)$ & & \\
\hline$>2-5$ & $13(16.7)$ & $5(6.9)$ & & \\
\hline \multicolumn{5}{|c|}{$\begin{array}{l}\text { Respiratory rate } \\
\text { (per minute) }\end{array}$} \\
\hline$<60 /$ minute & 60 (61.9) & $37(38.1)$ & 10.68 & $\mathrm{p}=0.001^{* * *}$ \\
\hline$>60 /$ minute & $18(34.0)$ & $35(66.0)$ & & \\
\hline \multicolumn{5}{|l|}{$\begin{array}{l}\text { Type of } \\
\text { pneumonia }\end{array}$} \\
\hline Pneumonia & $43(78.2)$ & $12(21.8)$ & 26.66 & $p=0.001^{* * *}$ \\
\hline $\begin{array}{l}\text { Severe } \\
\text { pneumonia }\end{array}$ & 25 (43.9) & $32(56.1)$ & & \\
\hline $\begin{array}{l}\text { Very severe } \\
\text { pneumonia }\end{array}$ & $10(26.3)$ & $28(73.7)$ & & \\
\hline
\end{tabular}

***The association was highly/strongly significant at the 0.006 and 0.001 levels of significance. The parenthesis () shows the corresponding percentage 
Grunting, nasal flaring subcostal and intercostal retractions were the factors that impacted strongly the hypoxemia but the suprasternal retraction influenced significantly. Grunting and nasal flaring were significantly associated $(\mathrm{p}=0.009, \mathrm{p}=0.008)$ with hypoxemia. Grunting was less sensitive $(16.66 \%)$ but highly specific $(96.15 \%)$ sign while nasal flaring was fairly specific (79.48) and sensitive $(40.27 \%)$ sign. Suprasternal retraction was significantly $(\mathrm{p}=0.024)$ associated with hypoxemia reported in $40(55.6 \%)$ hypoxemic children was fairly sensitive (55.55\%) and specific $(62.82 \%)$. Subcostal and intercostal retractions were found to be strongly associated with hypoxemia as the differences in groups were highly significant $(\mathrm{p}=0.001$ and $\mathrm{p}=0.000)$ on statistical ground. Subcostal retraction had high sensitivity $(81.94 \%)$ and fair specificity $(43.58 \%)$ but Intercostal retraction had a sensitive $(83.33 \%)$ and a fairly specific (44.87\%) sign (Table 3).

Table 4 reflects that all children had fever except 1.3\% non-hypoxemia children. Fever was very sensitive (100\%) but a nonspecific $(1.2 \%)$ symptom. Cough was a constant feature and it was present in all children. Dyspnea was diagnosed in most $(97.2 \%)$ of the hypoxemic children was very sensitive $(97.22 \%)$ but nonspecific $(7.6 \%)$ symptom while chest pain was present in $2.60 \%$ non-hypoxemic children wasn't a sensitive symptom. $15.4 \%$ non-hypoxemic and $25.0 \%$ hypoxemic children weren't accepted feeding normally and complained of decrease feeding since feeding was highly specific $(84.61 \%)$ but a less sensitive $(25 \%)$ symptom. The differences in groups for fever $(\mathrm{p}=0.335)$, dyspnea $(p=0.181)$, chest pain $(p=0.171)$ and decrease feeding $(p=0.141)$ couldn't reach at statistical significance.

Exactly one third $(33.3 \%)$ hypoxemic children were lethargic, $16.7 \%$ irritable and no hypoxemic child was drowsy while $38.9 \%$ lethargic, $16.7 \%$ irritable and $2.8 \%$ were drowsy hadn't hypoxemia and these differences in behavior couldn't reach at statistical significance $(p=0.384)$ because behavior was not recorded as the factor that influenced the hypoxemia. Drowsiness was most specific $(100 \%)$ but had low sensitivity $(2.5 \%)$. Irritability was also highly specific $(83.33 \%)$ and less sensitive $(16.66 \%)$ symptom while lethargy was fairly sensitive $(38.88 \%)$ and specific (58.97\%) symptom (Table 4).

\section{DISCUSSION}

This article offers an introduction to the understanding of determinants of hypoxemia among children diagnosed with pneumonia and the associating factors affected the healing process during pneumonic traumas that impacted adversely on condition of child health. Study reported that the average ages of non-hypoxemic and hypoxemic

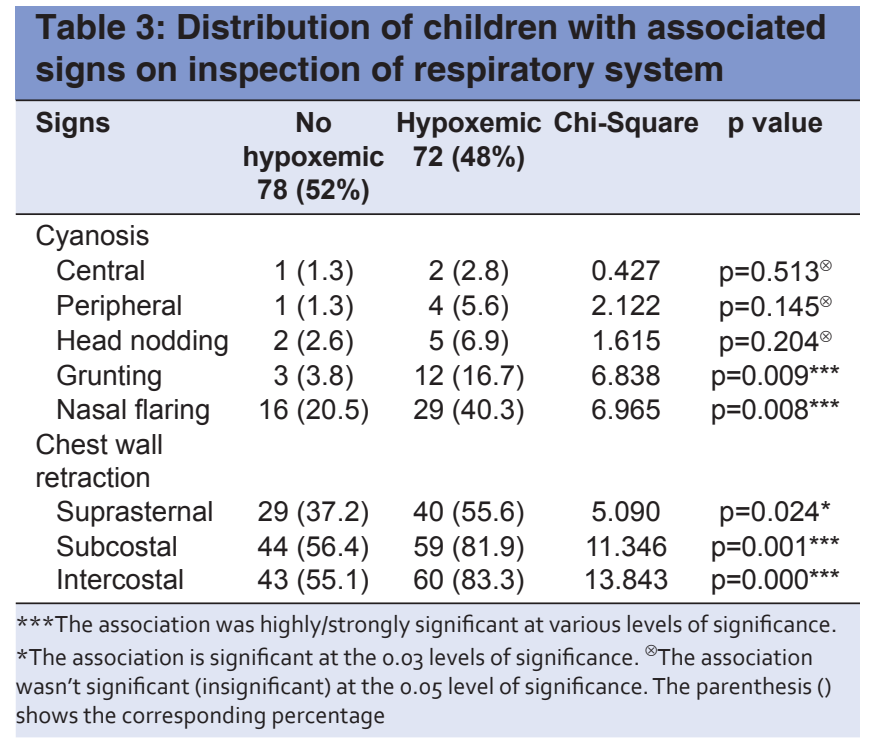

\begin{tabular}{|c|c|c|c|c|}
\hline Symptoms & $\begin{array}{c}\text { No hypoxemic } \\
78(52 \%)\end{array}$ & $\begin{array}{c}\text { Hypoxemic } \\
72(48 \%)\end{array}$ & Chi-Square & $p$ value \\
\hline Fever & $77(98.7)$ & $72(100.0)$ & 0.929 & $\mathrm{p}=0.335^{\otimes}$ \\
\hline Cough & $78(100)$ & $72(100)$ & NA & $N A^{\otimes}$ \\
\hline Dyspnea & 72 (92.3) & $70(97.2)$ & 1.791 & $\mathrm{p}=0.181^{\otimes}$ \\
\hline Chest pain & $2(2.6)$ & $0(0.0)$ & 1.871 & $\mathrm{p}=0.171^{\otimes}$ \\
\hline $\begin{array}{l}\text { Decrease } \\
\text { feeding }\end{array}$ & $12(15.4)$ & $18(25.0)$ & 2.163 & $\mathrm{p}=0.141^{\otimes}$ \\
\hline \multicolumn{5}{|l|}{ Behavior } \\
\hline Drowsy & $0(0.0)$ & $2(2.8)$ & 3.053 & $\mathrm{p}=0.384^{\otimes}$ \\
\hline Irritable & $13(16.7)$ & $12(16.7)$ & & \\
\hline Lethargy & $26(33.3)$ & 28 (38.9) & & \\
\hline Normal & $39(50)$ & $33(45.83)$ & & \\
\hline
\end{tabular}

${ }^{8}$ The association wasn't significant (insignificant) at the 0.05 level of significance. The parenthesis () shows the corresponding percentage. [NA-Not Applicable]

children were 15.44 and 10.44 months respectively and the differences in age were statistically significant which correlated with the finding of Lodha et al. (2004). ${ }^{14}$ The prevalence of hypoxemia documented in various studies with a variation. The present study showed $48 \%$, the prevalence of hypoxemia was more ${ }^{14-17}$ when compared to earlier studies in India. There were $24.4 \%$ female and $75.6 \%$ male children found without hypoxemia as compared to $37.5 \%$ female and $62.5 \%$ male children had hypoxemia but the gender differences between groups were statistically insignificant which was supported by Motwani et al. $(2015)^{10}$ observed that the difference between male and females, as regarding to prevalence of hypoxemia was not significant.

WHO defines tachypnoea as respiratory rate of 50 or more for infants (2-12 months) and 40 or more for children (13-60 months) of age since tachypnoea is a reliable predictor of pneumonia. Various researches 
have used higher cut-off values than WHO guideline to show association of tachypnoea with hypoxemia. ${ }^{1,8,18}$ Respiratory rate of more than 60 per minute (tachypnea) was present in 34\% non-hypoxemic than $66 \%$ hypoxemic children and the difference was significantly associated with hypoxemia which is corroborated with Basnet et al. $(2006)^{18}$ reported that respiratory rate of 70 per minute was significantly associated with hypoxemia while Lozano et al. $(1993)^{19}$ used respiratory rate of 50 per minute in their study to show association of tachypnoea with hypoxemia at high altitude. In present study, it was noted that hypoxemia was significantly associated with age was supported by Basnet et al., revealed that increased chances of pneumonia and frequency of hypoxemia in infants.

Present study showed that hypoxemia was significantly impacted and strongly associated with the severity of pneumonia and $21.8 \%, 56.1 \%$ and $2873.7 \%$ hypoxemicchildren respectively had non severe, severe and very severe pneumonia which is in agreement with the study that demonstrated a significant association of severity of illness with CFR ranging from $0-47 \%$ in children with pneumonia, severe pneumonia, and very severe pneumonia as per WHO classification. ${ }^{20}$ It is difficult to understand the practical importance of this association because the clinical course and pathway to death can't be visualized if the cases are not categorized based on severity of illness at the time of admission ${ }^{13}$ while Kabara et al. ${ }^{21}$ evaluated that clinical signs alone or in combination do not have sufficient sensitivity and specificity to predict hypoxemia in children with pneumonia.

In a recent research, Motwani et al. $(2015)^{10}$ reported that chest wall retractions and crepitation had fair sensitivity. WHO criteria for giving $\mathrm{O}_{2}$ to children with pneumonia are inability to feed/drink, central cyanosis, grunting, chest in-drawing, head nodding or respiratory rate of 70 per minute or above. ${ }^{22}$ The research has shown that the central cyanosis $(98.7 \%)$, peripheral $(98.7 \%)$ cyanosis, head nodding $(97.4 \%)$ grunting $(96.15 \%)$ were highly specific but all were less sensitive sign but suprasternal $(62.82 \%)$, subcostal $(43.58 \%)$ and intercostal retraction $(44.87 \%)$ were fairly specific sign. Sensitivity for subcostal $(81.94 \%)$ and intercostal retractions $(83.33 \%)$ was very high but was fair for intercostal retraction $(83.33 \%)$. Similar results were replicated in various reports as cyanosis, head nodding, drowsiness; respiratory rate more than 60 per minute, grunting and nasal flaring was seen in a study by Onyango et al. to be significantly associated with hypoxemia ${ }^{8}$ while Sah highlighted different predictors for hypoxemia were inability to feed and drink, lethargy, tacypnoea, central cyanosis, chest in-drawing and grunting. ${ }^{12}$
Findings of the study revealed that grunting $(\mathrm{p}=0.009)$, nasal flaring $(p=0.008)$, subcostal $(p=0.001)$ and intercostal $(\mathrm{p}=0.000)$ retractions were highly but suprasternal retraction was significantly $(\mathrm{p}=0.024)$ associated with hypoxemia which is in agreement with various other studies have reported that grunting and nasal flaring were significantly associated with hypoxemia. ${ }^{1,18,23}$ During a study, Rao et al. $(2012)^{16}$ found that chest wall retraction was found to be the most sensitive indicator, and cyanosis was the most specific indicator for hypoxemia. Kuti has also confirmed the relevance of the study as he recently reported that children that grunt and are centrally cyanosed should preferentially commenced on oxygen therapy even when there is no facility to confirm hypoxemia. ${ }^{24}$ Further, present research showed that fever and cough were constant features while dyspnea was very sensitive ( $97.22 \%$ ) but feeding was highly specific (84.61\%) symptom. Irritability $(83.33 \%)$ was highly but lethargy $(58.97 \%)$ was fairly specific symptom.

Various findings replicated in the research conducted by Sah, found that among clinical signs lethargy, central cyanosis, nasal flaring, grunting, chest in-drawing, tachypnea and crept were found to be significantly associated with hypoxemia. ${ }^{12}$ Agrawal K et al. (2011) reported that infants presenting with $\geq 3$ symptoms, lethargy, respiratory rate of $\geq 70 /$ minute or chest in-drawing can be used for screening purpose to detect hypoxia and infants showing signs like grunting, head nodding, nasal flaring or central cyanosis should be considered hypoxic and treated with supplemental oxygen. ${ }^{25}$

Overall, the article has a view in support of the early detection and effective management of hypoxia is an important aspect of the clinical management in infant and child with pneumonia. An integrated approach by undertaking the signs and symptoms recorded for pneumonia in-depth would ensure the reduction in length of illness and hospitalization associated to pneumonic trauma and a more effective way of rehabilitation and promotion of pediatric sciences.

\section{CONCLUSIONS}

Prevention and treatment require more clinical and research attention to reduce the public health burden of pneumonia among hypoxemic children. The understanding of the nature and causes of pneumonic trauma has evolved over the centuries, though this understanding is incomplete and has left many aspects of hypoxia with pneumonia with as the subject of discussion and research. This study has helped in identifying certain signs and symptoms in pneumonia that can predict and associate with hypoxia and which can be best determined 
by the use of a pulse oxymeter but unfortunately in many centers it isn't available. Therefore, simple clinical signs and symptoms could learnt by health professionals to be used to diagnose hypoxemic children with pneumonia to provide oxygen therapy since hypoxemiaassociated severity of pneumonia would require oxygen administration as part of treatment.

Authors strongly support the introduction of a routinely determination of hypoxia for pneumonic children in order to begin as soon as possible an adequate treatment. Overall, this research identified determinants of hypoxemia in children associated with pneumonia that can predict hypoxia and hence health professionals may provide necessary treatment.

\section{Implications of the study}

More risk noted in pneumonic children for hypoxemia. Grunting, nasal flaring, decrease feed, dyspnea, subcostal and intercostal retractions were the significant factors that have to consider by a health professional with more precautions while treating hypoxemia among children. Hypoxia is probably very common in pneumonic children and has significant effects on important functional outcomes.

An early detection of hypoxia followed by and adequate intervention based on oxygen support can provide good results and better functional recovery among pneumonic children. In brief, the study-evaluated determinants of hypoxemia in children associated with pneumonia responsible for better recovery after hypoxic-illness and therefore awareness among medical professionals regarding the hypoxic-illness during pneumonic trauma may create.

\section{ACKNOWLEDGMENTS}

Authors are grateful to the Dr. Praveena Dhiman, Senior consultant of Pediatrics department at Government MultiSpeciality Hospital, Chandigarh for her kind support and valuable suggestions during research study.

\section{REFERENCES}

1. Usen S, Weber M, Mullholland K, Jaffar S, Oparaugo A and Omosigho C. Clinical predictors of hypoxaemia in Gambian children with acute lower respiratory tract infection: prospective cohart study. BMJ 1999; 318:86-91.

2. Denny FW and Loda FA. Acute respiratory infections are the leading cause of death in children in developing countries. Am J Trop Med Hyg 1986; 35(1):1-2.

3. Anthony GS, Scott W, Abdullah WB, Malik JS and Holtzman D. Pneumonia research to reduce childhood mortality in the developing world. J Cin Invest 2008; 118:1291-1300.
4. Theodore C, Sectish and Charles GP. Chapter 397, Pneumonia; Nelson Textbook of Pediatrics, $16^{\text {th }}$ Addition, Published 2007:1795.

5. Park K. Textbook of Preventive and Social Medicine. $19^{\text {th }}$ Addition, Published-2007; 452-458.

6. Mower WR, Sachs C, Nicklin EL and Baraff LJ. Pulse oximetry as a fifth vital sign, Pediatrics 1997; 99:681-686.

7. Schnapp LM and Cohen NH. Pulse oximetry: uses and abuses. Chest 1990; 98:1244-1250.

8. Onyango FE, Steinhoff MC, Wafula EM, Wariua S, Musia J and Kitonyi J. Hypoxemia in young Kenyan children with acute lower respiratory tract infection. BMJ 1993; 306: 612-614.

9. World Health Organization programme for the control of acute respiratory infections. Acute respiratory infections in children: case management in small hospitals in developing countries. Geneva: WHO/ARI/90.5.1990.

10. Motwani NP, Janakiraman LB, Subramanian S and Bhawnani D. Clinical predictors of hypoxemia in children with acute lower respiratory illness. Int Res J Medical Sci 2015; 3(3):11-15.

11. Lozano JM. Epidemiology of hypoxaemia in children with acute lower respiratory infection: Review study. Int J Tuberc Lung Dis 2001; 5:496-504.

12. Sah HD. Study of predictors of hypoxemia in children with Pneumonia. J Coll Med Sci Nepal 2013; 9(4):1-8.

13. Patwari AK. Risk factors for mortality in children hospitalized with Pneumonia. Indian Pediatr 2012; 49:869-870.

14. Lodha R, Bhaduaria PS, Kuttikat AV, Puranik M, Gupta S, Pandey RM et al. Can clinical signs or symptoms accurately predict hypoxemia in children with acute lower respiratory tract infections? Indian Pediatr 2004; 41:129-135.

15. Singhi S, Deep A and Kaur H. Prevalence and predictors of hypoxia in acute respiratory infections presenting to pediatric emergency department. IJCCM 2003; 7(11):118-123.

16. Rao YK, Midha T, Kumar P, Tripathi VN and Rai OP. Clinical predictors of hypoxemia in Indian children with acute respiratory tract infection presenting to pediatric emergency department. World J Pedr 2012; 8(3):247-51.

17. Rajesh VT, Singhi $S$ and Kataria S. Tachypnea is a good predictor of hypoxia in acutely ill children. Arch Dis Child 2000; 82:46-49.

18. Basnet S, Adhikari RK and Gurung CK. Hypoxemia in children with pneumonia and its clinical predictors. Indian J Pediatr 2006; 73(9):777-781.

19. Lozano JM, Steinhoff M, Ruiz JG, Mesa ML, Martinez N and Dussan B. Clinical predictors of acute radiological pneumonia and hypoxemia in young children in Bogota. Int Soc Technol Asses Health Care Meet 1993; 9:23.

20. Sehgal V, Sethi GR, Sachdev HP and Satyanarayana L. Predictors of mortality in subjects hospitalized with acute lower respiratory tract infections. Indian Pediatr 1997; 34: 213-9.

21. Kabra SK, Lodha R, Bhadauria PS, Kuttikat AV, Puranic M, Gupta $S$ et al. Can clinical symptoms or signs accurately predict hypoxemia in children with acute lower respiratory tract infection. Indian Pediatr 2004; 41:129-135.

22. Oxygen therapy. Management of the child with a severe infection or severe malnutrition - Guidelines for care at the first referral level in developing countries. (draft 25, 9/11/1998) World Health Organization, page 175.

23. Weber MW, Usen S, Palmer A, Jaffar S and Mulholland EK. Predictors of hypoxemia in hospital admission with acute respiratory tract infection in a developing country. Arch Dis Child 1997; 76(4):310-314.

24. Kuti BP, Adegoke SA, Ebruke BE, Howie S, Oyelami OA and 
Ota M. Determinants of oxygen therapy in childhood pneumonia in a resource-constrained region. ISRN Pedia 2013: Article ID 435976:6 pages.
25. Agrawal K, Mahaseth $\mathrm{C}$ and Rayamajhi A. Relationship of Respiratory Symptoms and Signs with Hypoxemia in Infants under 2 months of Age. J Nepal Paediatr Soc 2011; 31(3):202-208.

Authors Contributions:

PR- Concept, collected data, design of the study, literature search, manuscript preparation, prepared first draft of manuscript and critical revision of the

manuscript. BKS- Conceptualized study, reviewed the literature, statistically analysed, interpreted, reviewed first draft of manuscript and critical revision of the

manuscript. 